\title{
Condensin Depletion Causes Genome Decompaction Without Altering the Level of Global Gene Expression in Saccharomyces cerevisiae
}

\author{
Matthew Robert Paul,*,t Tovah Elise Markowitz, * Andreas Hochwagen, *,1 and Sevinç Ercan*,t,1 \\ *Department of Biology and ${ }^{\dagger}$ Center for Genomics and Systems Biology, New York University, New York 10003 \\ ORCID IDs: 0000-0002-3020-7729 (M.R.P.); 0000-0002-6184-443X (A.H.); 0000-0001-7297-1648 (S.E.)
}

\begin{abstract}
Condensins are broadly conserved chromosome organizers that function in chromatin compaction and transcriptional regulation, but to what extent these two functions are linked has remained unclear. Here, we analyzed the effect of condensin inactivation on genome compaction and global gene expression in the yeast Saccharomyces cerevisiae by performing spike-incontrolled genome-wide chromosome conformation capture (3C-seq) and mRNA-sequencing analysis. 3C-seq analysis shows that acute condensin inactivation leads to a global decrease in close-range intrachromosomal interactions as well as more specific losses of interchromosomal tRNA gene clustering. In addition, a condensin-rich interaction domain between the ribosomal DNA and the centromere on chromosome XII is lost upon condensin inactivation. Unexpectedly, these large-scale changes in chromosome architecture are not associated with global changes in mRNA levels. Our data suggest that the global transcriptional program of proliferating $S$. cerevisiae is resistant to condensin inactivation and the associated profound changes in genome organization.
\end{abstract}

KEYWORDS genome organization; budding yeast; condensin; chromosome interactions; gene expression; TADs

RGANIZED compaction of eukaryotic genomes regulates important nuclear processes including transcription, replication, and repair (Dekker and Mirny 2016; Dixon et al. 2016). Classic examples of nuclear organization include compartmentalization of transcriptionally active and repressed domains, as well as specialized structures as in the segregation of the ribosomal DNA (rDNA) into the nucleolus (Heitz 1928; Edstrom et al. 1961; Frenster et al. 1963). Recent measurements of pairwise interaction frequencies between chromosomal sites revealed additional structural elements, including stable topologically associating domains (TAD) on the scale of 0.1-1 Mb (Dixon et al. 2012, 2015; Sexton et al. 2012), but also smaller more dynamic interaction domains linked to gene regulation (Jin et al. 2013; Rao et al. 2014; Rocha et al. 2015; Ji et al. 2016). Restriction of chromosomal interactions within TADs is thought to support

Copyright (c) 2018 by the Genetics Society of America doi: https://doi.org/10.1534/genetics.118.301217

Manuscript received June 4, 2018; accepted for publication June 25, 2018; published Early Online July 3, 2018.

Supplemental material available at Figshare: https://figshare.com/s/f13d3897a68f4 f8e4510.

${ }^{1}$ Corresponding authors: Department of Biology, New York University, 1009 Silver Center, 100 Washington Square East, New York, NY 10003-6688. E-mail: andi@ nyu.edu; and se71@nyu.edu enhancer-promoter specificity and is important for normal development (Lettice et al. 2011; Ibn-Salem et al. 2014; Giorgio et al. 2015; Lupiáñez et al. 2015).

The evolutionarily conserved condensins have emerged as major regulators of chromosomal architecture. Condensins belong to the structural maintenance of chromosomes (SMC) family of chromosome organizers, which also includes cohesin and the SMC5/6 complex (Uhlmann 2016; van Ruiten and Rowland 2018). Like other SMC complexes, condensin forms a ring-like structure and topologically encircles DNA to mediate chromosomal looping (Cuylen et al. 2013; Gligoris and Lowe 2016). Condensins are essential for chromosome condensation and segregation during cell division, and have been implicated in several types of chromosomal interactions during mitosis, including lateral and axial compaction, as well as site-specific interactions (Kinoshita and Hirano 2017). The scale of these interactions differs somewhat between species and involves two distinct condensin complexes in metazoans (Gibcus et al. 2017; Kakui et al. 2017; Schalbetter et al. 2017).

In addition to mitotic chromosome structure, condensin complexes also regulate nuclear organization in interphase in a number of organisms (D'Ambrosio et al. 2008; Kranz et al. 
2013; Nishide and Hirano 2014; Crane et al. 2015; Kschonsak and Haering 2015; Leonard et al. 2015; Nakazawa et al. 2015; Sutani et al. 2015; X. Wang et al. 2015; Hirano 2016). Interphase activities include the organization of TADs on specific chromosomes or across the genome (Crane et al. 2015; Li et al. 2015), and the regulation of interchromosomal interactions. For example, condensin complexes control X chromosome organization in Caenorhabditis elegans (Crane et al. 2015), chromosome pairing in Drosophila melanogaster (Hartl et al. 2008), and the clustering of cell cycle-regulated genes in Schizosaccharomyces pombe (Kim et al. 2016). In Saccharomyces cerevisiae, condensin is required to tether the centromere of chromosome (Chr) XII to the ribosomal DNA (rDNA), rDNA compaction upon starvation, and tRNA gene clustering (Haeusler et al. 2008; Wang et al. 2016; Lazar-Stefanita et al. 2017; Schalbetter et al. 2017). Many of these specific condensin-mediated interactions have been shown to have important functions in gene regulation (Iwasaki et al. 2010; Crane et al. 2015). It remains to be established if the general genome compaction by condensin similarly results in global effects on gene expression.

In this study, we addressed the relationship between condensin-mediated chromatin compaction and global gene expression in $S$. cerevisiae using genome-wide chromosome conformation capture (3C-seq) and mRNA-sequencing (mRNA-seq) when condensin function is disrupted in logarithmically growing cells. We find that loss of condensin function leads to a shift from shorter- to longer-range chromosomal interactions and a loss of locus-specific interactions involving tRNA genes as well as the rDNA. Intriguingly, these architectural changes were not associated with broad changes in global gene expression levels. These data suggest that the structural changes associated with condensin depletion are not directly coupled to transcription.

\section{Materials and Methods}

\section{Cell culture and preparation of nuclei}

Inducible nuclear depletion of condensin was achieved using the anchor-away approach (Haruki et al. 2008), specifically by C-terminally tagging the condensin subunit Brn1 in the SK1 background with an FKBP-Rapamycin binding (FRB) domain. Asynchronous cells were grown in $200 \mathrm{ml}$ of YPD to $\mathrm{OD} 1$ at $30^{\circ}$. Condensin depletion was induced using rapamycin at $1 \mu \mathrm{g} \cdot \mathrm{ml}^{-1}$ for $30 \mathrm{~min}$. Temperature-sensitive condensin mutants harbor the $y c s 4-2$ or ycg1-2 allele in the SK1 background (Lavoie et al. 2002; Yu and Koshland 2003). Wild-type and mutant yeast cells were grown asynchronously at permissive temperature $\left(23^{\circ}\right)$ until OD1, then shifted to restrictive temperature $\left(37^{\circ}\right)$, or kept at permissive temperature, for $2 \mathrm{hr}$. For 3C-seq experiments, yeast cells were fixed for $30 \mathrm{~min}$ at room temperature by adding formaldehyde to the media at 3\% and quenching using $125 \mathrm{mM}$ glycine. Spheroplasts were prepared by digesting the cell wall with zymolyase (Amsbio, Cambridge, MA) and then resuspending in dounce buffer (0.35 $\mathrm{M}$ sucrose, $15 \mathrm{mM}$ HEPES $\mathrm{KOH}$, $0.5 \mathrm{mM}$ EGTA, $5 \mathrm{mM} \mathrm{MgCl}_{2}, 10 \mathrm{mM} \mathrm{KCl}, 0.1 \mathrm{mM}$ EDTA, $0.5 \%$ Triton $\mathrm{X}-100$, and $0.25 \%$ NP40). Nuclei were released using a type B dounce and glass pestle on ice. Cellular debris was removed by spinning at $230 \times g$. The supernatant was spun down at $2000 \times g$ to isolate the nuclei. Nuclei were stained with the vital dye Crystal violet in citric acid (Fisher Scientific, Pittsburgh, PA) to allow counting on a hemocytometer as described previously (Spearman 2007).

\section{C-seq}

For the anchor-away samples, 3C-seq was used as previously described (Sexton et al. 2012). Around 100 million nuclei were permeabilized by incubating in $500 \mu \mathrm{l}$ NEB restriction enzyme buffer 2.1 (New England Biolabs, Beverly, MA) with $0.3 \%$ SDS for $1 \mathrm{hr}$ at $37^{\circ}$ in a thermomixer. SDS was quenched by adding $2 \%$ Triton X-100, and nuclei were incubated for $1 \mathrm{hr}$ further at $37^{\circ}$ in a thermomixer. Chromatin was digested using 1200 units of restriction enzyme HindIII (New England Biolabs) overnight at $37^{\circ}$ in a water bath. Following digestion, the volume was increased to a total of $8 \mathrm{ml}$ using $1 \times$ T4 DNA Ligase Buffer (New England Biolabs). Chromatin fragments were ligated with 750 units of T4 DNA ligase overnight at $15^{\circ}$ (Thermo Fisher Scientific, Waltham, MA). For the temperature-sensitive samples, in situ 3C-seq was performed based on two protocols (Lieberman-Aiden et al. 2009; Sexton et al. 2012). Briefly, nuclei were permeabilized by incubating in $50 \mu \mathrm{l}$ of $0.5 \%$ SDS for $10 \mathrm{~min}$ at $62^{\circ}$ in a thermomixer. SDS was quenched by adding $170 \mu \mathrm{l}$ of $1.5 \%$ Triton X-100, and nuclei were incubated for $15 \mathrm{~min}$ at $37^{\circ}$ in a thermomixer. Next, $25 \mu$ l of $10 \times$ NEB restriction enzyme buffer 2.1 was added and chromatin was digested using 1200 units of restriction enzyme HindIII overnight at $37^{\circ}$ in a water bath. Following digestion, the volume was increased to a total of $1.15 \mathrm{ml}$ using $1 \times$ T4 DNA Ligase Buffer (New England Biolabs). Chromatin fragments were ligated with 150 units of T4 DNA ligase overnight at $15^{\circ}$ (Thermo Fisher Scientific). 3C DNA was purified using QIAGEN PCR purification columns (QIAGEN, Valencia, CA). DNA was sheared using a Bioruptor Pico (Diagenode, Denville, NJ) by diluting $500 \mathrm{ng}$ of purified DNA in $50 \mu \mathrm{l}$ and sonicating with the following settings: $15 \mathrm{sec}$ on and $90 \mathrm{sec}$ off for seven cycles, with a quick vortex and spin down after four cycles. Libraries were prepared from the sonicated DNA by PCR following the addition of Illumina TruSeq adapters as described previously (Kranz et al. 2013). Sequencing was performed at the New York University Biology Genomics core using paired-end 50-bp sequencing on the Illumina HiSeq-2500.

\section{C-seq spike-in}

To a subset of samples, we spiked in $10 \%$ C. elegans nuclei prior to continuing with the 3C-seq protocol above. C. elegans $\mathrm{N} 2$ embryos were washed with $500 \mu$ l of chitinase buffer (110 mM NaCl, $40 \mathrm{mM} \mathrm{KCl}, 2 \mathrm{mM} \mathrm{CaCl} 2,2 \mathrm{mM} \mathrm{MgCl}$, and $25 \mathrm{mM}$ HEPES-KOH pH 7.5) and digested with 5 units of chitinase (Sigma [Sigma Chemical], St. Louis, MO). The 
resulting blastomeres were spun-down at $2000 \times g$ for $5 \mathrm{~min}$ at $4^{\circ}$, then resuspended in PBS with $1 \%$ formaldehyde and fixed for 30 min while nutating. Formaldehyde was quenched with $125 \mathrm{mM}$ glycine. Blastomeres were washed and nuclei released in dounce buffer (see yeast nuclei preparation). Cellular debris was removed by spinning at $100 \times g$. The supernatant was spun at $2000 \times g$ to isolate the nuclei.

\section{C-seq data analysis}

The data statistics, such as read number and mapping efficiency, for each replicate are given in Supplemental Material, File S1. Data were processed using previously described methods, using the Mirny laboratory Python packages (https://bitbucket.org/mirnylab/) (Imakaev et al. 2012). Briefly, iterative mapping was used to map each side of the paired-end read individually. For the spike-in experiments, the reads that uniquely mapped to either $C$. elegans (WS220) or S. cerevisiae (SacCer3) were filtered and used separately for each species. Reads were then assigned to the closest restriction fragment end. Counts were binned into 10-kb bins. Default filters were applied to remove outlier regions such as those with poor coverage (bottom $1 \%$ or with $<50 \%$ of bin sequenced) or contain PCR duplicates (top $0.05 \%$ ). Iterative correction was used to correct for potential biases including mappability and GC content, without predefining what these biases are.

\section{Chromatin immunoprecipitation-sequencing and data analysis}

Chromatin immunoprecipitation (ChIP) was performed as described (Blitzblau et al. 2012). Samples were immunoprecipitated with $20 \mu \mathrm{l}$ anti-V5 agarose affinity gel (Sigma). ChIP and input DNA was resonicated using a Bioruptor Pico (Diagenode) with the following settings: $30 \mathrm{sec}$ on and $30 \mathrm{sec}$ off for five cycles. Libraries were prepared by PCR following the addition of Illumina TruSeq adapters as described previously (Kranz et al. 2013). Sequencing was performed using 50-bp single-end sequencing on an Illumina HiSeq 2500 instrument at the New York University Biology Genomics core. Sequencing reads were mapped to the SK1 genome (Yue et al. 2017) using Bowtie (Langmead et al. 2009). Reads that mapped to only one location without mismatches were used in further analyses. Further processing was completed using MACS-2.1.0 (https://github.com/taoliu/MACS) (Zhang et al. 2008). Reads were extended toward $3^{\prime}$ ends to a final length of $200 \mathrm{bp}$ and probabilistically determined PCR duplications were removed. Pileups of both the input and ChIP libraries were signal per million reads-normalized, followed by a calculation of the foldenrichment of the ChIP data over the input data, resulting in the ChIP-coverage scores that are shown in the figures.

\section{mRNA-seq with S. pombe spike-in}

For spike-in, $4.5 \mathrm{ml}$ of OD1 S. cerevisiae was mixed with $500 \mu \mathrm{l}$ of $S$. pombe $(972 \mathrm{~h})$ at OD1. To ensure that the ratio of $S$. pombe to $S$. cerevisiae was consistent between samples, genomic DNA was isolated from $10 \%$ of the mixed cells and used for quantitative PCR (qPCR). Briefly, serial dilutions of $S$. pombe and $S$. cerevisiae were amplified using both $S$. pombeand $S$. cerevisiae-specific primers (File S1) to generate standard curves, to which the amplification from experimental samples was compared. qPCR-measured ratios of spike-in to experimental sample ranged between $\sim 10 \% \pm 2$. The cells in the remaining 90\% were broken with glass beads and RNA was purified over a QIAGEN RNeasy column following the recommended yeast protocol. mRNA was selected using Sera-Mag oligo-dT beads (GE Healthcare Life Sciences, Marlborough, MA) from 0.5 to $10 \mu \mathrm{g}$ of total mRNA. cDNA was prepared based on the incorporation of deoxyuridine triphosphates during cDNA synthesis using a previously described protocol (Parkhomchuk et al. 2009). Libraries were prepared from the cDNA by PCR following the addition of Illumina TruSeq adapters (Kranz et al. 2013). Sequencing was performed at the New York University Biology Genomics core using single-end 50-bp sequencing on the Illumina HiSeq-2500.

\section{mRNA-seq analysis with S. pombe spike-in}

S. pombe (ASM294v2) and S. cerevisiae (SacCer3) were mapped using TopHat version 2.0.11 (Trapnell et al. 2009) and reads mapping to both genomes were discarded from further analyses. Raw counts for each gene were determined using htseq-count version 0.6.1 (Anders et al. 2015) and normalized based on total reads. Correction of $S$. cerevisiae based on $S$. pombe spike-in was done as previously published (Kramer et al. 2015). Briefly, a linear model was fitted to $S$. pombe spike-in data, comparing read counts of expressed genes (counts per million $>0.5$ ) in the wild-type to other experimental conditions. The coefficients from this linear model were then used to correct the $S$. cerevisiae expression values. Clustering was performed in $\mathrm{R}$ version 3.3 .2 with hierarchical clustering using the complete linkage model. Dendrograms were cut to produce four clusters, as predicted by the elbow method, silhouette analysis, and the Calinksi criterion.

\section{Data availability}

All data sets used in this publication, including processed files, can be found on the Gene Expression Omnibus series GSE106104. Individual sample indexes can be found in File S1. Scripts and code used for analysis of this data are deposited at https://github.com/ercanlab. Supplemental material available at Figshare: https://figshare.com/s/f13d3897a68f4f8e4510.

\section{Results}

To investigate the link between chromosome structure and gene expression, we performed 3C-seq analysis, which captures the frequency of pairwise contacts between genomic loci (Sexton et al. 2012; Crane et al. 2015), and mRNA-seq analysis in S. cerevisiae (yeast) upon condensin perturbation. Unlike metazoans, yeast contain a single condensin complex (Figure 1A). We acutely removed condensin from yeast nuclei using the inducible anchor-away system targeted against the kleisin subunit Brn1. In this system, proteins tagged with 
the FRB domain of human TOR kinase can be conditionally depleted from the nucleus by the addition of rapamycin (Figure 1B) (Haruki et al. 2008). Validating this approach, the parental anchor-away strain was capable of growing on rapamycin plates whereas a strain containing FRB-tagged Brn1 was not (Figure S1A). Nuclear depletion of condensin was confirmed by imaging the condensin subunit Smc4 tagged with GFP (Figure S1B). In an independent approach, we used a strain bearing a temperature-sensitive mutation in the Ycs4 subunit $(y c s 4-2)$ to conditionally inactivate condensin by shifting cells to the nonpermissive temperature of $37^{\circ}$ (Lavoie et al. 2002). Sequencing showed that this allele harbors two mutations, which change amino acids 219 (methionine to lysine) and 225 (cysteine to arginine) of Ycs4 (Figure 1C). In both approaches, the treatments did not trigger enrichment of cells at a specific cell cycle stage (Figure S1C).

\section{Shift from short- to longer-range chromosomal interactions in the absence of condensin}

Plotting normalized interaction frequencies between 10-kb bins of the wild-type data set showed many of the features common to chromosome conformation capture experiments in yeast and other organisms (Duan et al. 2010; Rutledge et al. 2015). Interactions between loci on the same chromosome are more frequent compared to trans interactions (Figure 1D). Moreover, distance-decay curves show that such cis interaction frequencies decrease as the genomic distance between loci increases (Figure 1E). Condensin depletion led to a reduction in local interactions with a marked increase in trans interactions and long-range cis interactions (Figure 1F). This result is also supported by the flattened distance decay curves (Figure 1E) and highlighted by plotting the z-score subtraction of interaction frequencies between Brn1depleted and wild-type cells (Figure $1 \mathrm{H}$ ). An increase in longer-range interactions at the expense of short-range $(<$ $100 \mathrm{~kb}$ ) ones was also observed in the ycs4-2 strain at the restrictive temperature (Figure $1 \mathrm{G}$ and Figure S1D). Our results in cycling cells agree with recent depletion experiments using an auxin-dependent degron on the condensin subunit Smc2 in mitosis (Lazar-Stefanita et al. 2017), whereas a temperature-dependent degron on Smc2 led to more limited effects (Schalbetter et al. 2017).

To ensure that the change in the range of chromosomal interactions upon condensin inactivation was not due to technical differences between the wild-type and mutant samples, we used a spike-in approach. We added C. elegans nuclei at similar proportions to wild-type and ycs 4-2 nuclei before proceeding with the 3C-seq protocol. Reads unique to each organism were then used independently to produce decay curves of interaction frequencies within each sample. The spike-in controls showed a similar pattern of decay for interaction frequencies in 3C-seq reactions containing wild-type and $y$ cs 4-2 mutant nuclei (Figure 1I), validating that condensin mutation causes a shift from short- to long-range interactions in yeast. These results are consistent with condensin promoting local compaction across the genome.

\section{Centromere and telomeres maintain clustering in the absence of condensin}

A characteristic feature of yeast genome organization is the tethered clustering of centromeres and telomeres in the nuclear periphery (Rabl conformation) (Gottschling et al. 1990; Palladino et al. 1993; Jin et al. 2000). These features have previously been observed through chromosome capture approaches (Duan et al. 2010; Tjong et al. 2012; Rutledge et al. 2015; R. Wang et al. 2015; Hsieh et al. 2016) and were also apparent in our data. In the heat maps, the strong trans interactions between centromeres were observed as an array of foci (Figure 1D). Moreover, nearly all of the 240 potential pairwise interactions between centromeres were in the top $1 \%$ of all interactions, with a significant enrichment compared to random shuffling of centromeres across the genome (Figure 2A). Following condensin depletion, centromere clustering was maintained as previously observed with immunofluorescence (Yong-Gonzalez et al. 2007), indicating that centromere attachment to the spindle pole is largely unaffected by condensin removal. However, our data do not exclude transient effects, as have been observed for centromere clustering at the metaphase-to-anaphase transition (Lazar-Stefanita et al. 2017).

We also observed significant enrichment of pairwise telomere interactions in wild-type cells, though the strength of clustering was weaker compared to centromeres (Figure 2B). Similar to centromeres, condensin depletion did not significantly alter telomere clustering in cycling cells (Figure 2B).

The condensin independence is likely due to the physical tethering of centromeres and telomeres to the nuclear periphery and does not reflect a general resistance of these regions to condensin-mediated effects. Accordingly, cis interactions of regions immediately flanking the centromeres were reduced upon condensin depletion similar to the rest of the genome (Figure S2, A and B). These data indicate that the clustering of centromeres and telomeres is refractory to the large changes in chromosomal interactions upon condensin depletion.

We asked whether condensin depletion affects the Rabl conformation by regulating the long-range interaction between the two arms of each chromosome. In wild-type cells, long-range interactions across chromosome arms are greater than interactions within one arm, suggesting that the Rabl conformation puts the arms in register. Upon condensin depletion, the interactions between the two arms of a single chromosome were reduced (Figure 2C), suggesting that the volume exclusion of chromatin that is essential to the Rabl conformation is strengthened by condensin, allowing chromosome arms to stay in contact.

\section{Condensin is required for clustering of a subset of tRNA genes}

Previous cytological analyses revealed condensin-dependent clustering and association of yeast tRNA genes with the nucleolus, the location of the rDNA (Haeusler et al. 2008; 
A

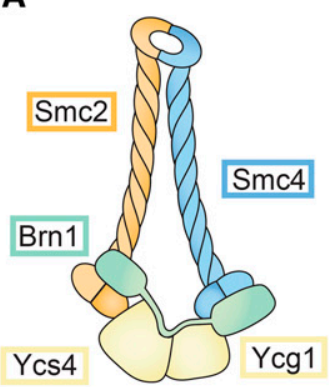

B

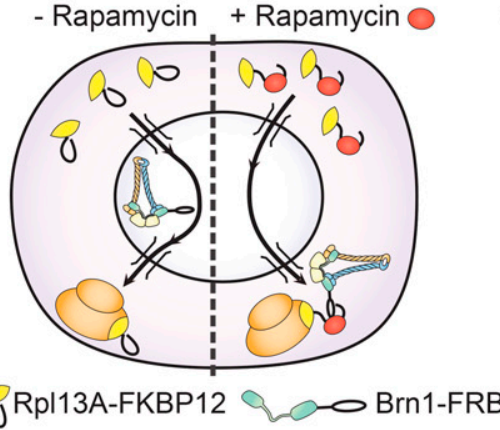

C

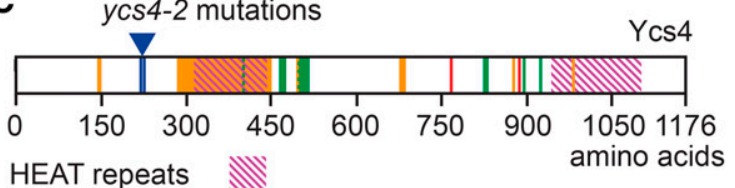

HEAT repeats

Brn1 binding

Ycg1 binding

Phosphorylation

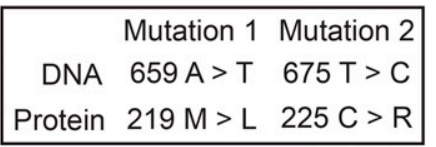

D

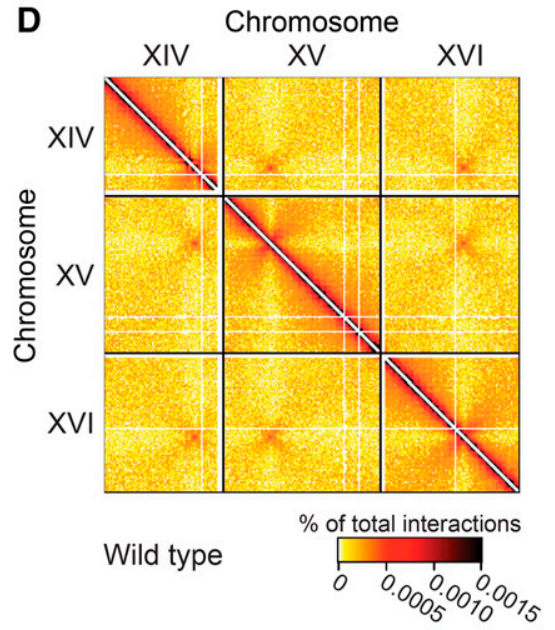

F

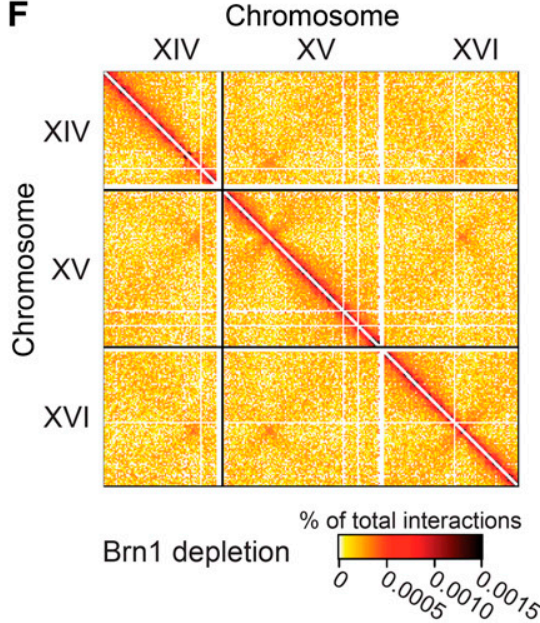

H

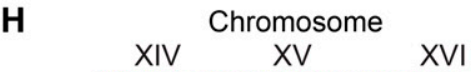

\section{E}

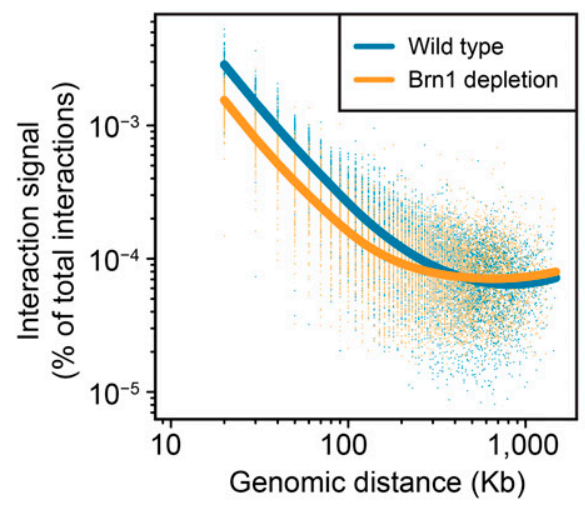

G

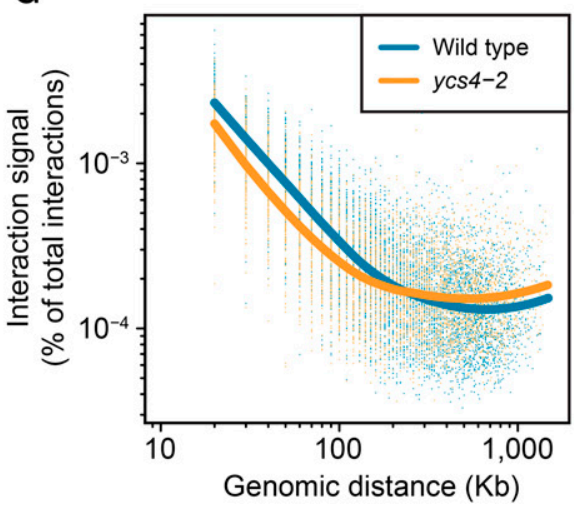

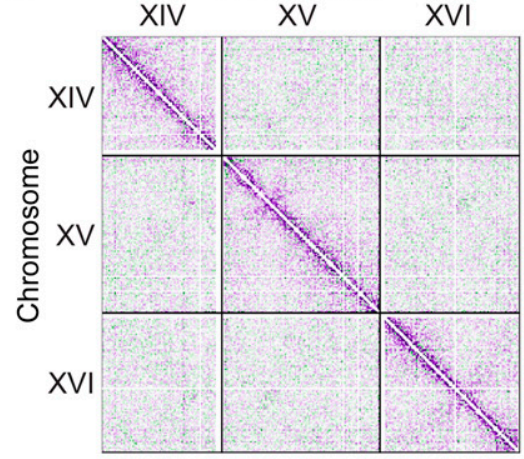

Wild z-score difference type $\quad \begin{array}{cccccc}-4 & -2 & 0 & 2 & 4\end{array}$
I

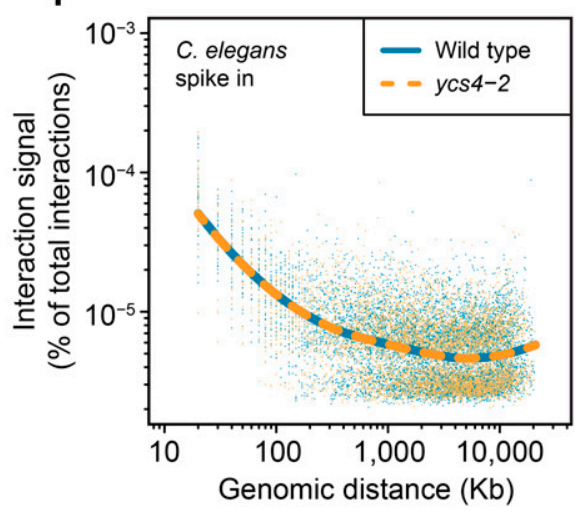

Figure 1 The $S$. cerevisiae genome is decompacted upon condensin perturbation. (A) Schematic of the five subunits that make up the condensin complex. (B) Schematic of the anchor-away approach. Upon addition of rapamycin, the FKBP-Rapamycin binding (FRB) domain fused to Brn1 binds to the FK506 binding protein 12 (FKBP12) domain fused to the ribosomal subunit Rpl13A. As a result, condensin is shuttled out of the nucleus. (C) The location of the nonsynonymous ycs4-2 mutations. Previously mapped structural and functional domains, and known phospho-acceptor sites are indicated. (D) Chromosome interaction frequencies across three large yeast chromosomes in wild-type (parental anchor-away strain). Both wild-type and Brn1 anchor-away strains were grown asynchronously in logarithmic phase and cells were collected $30 \mathrm{~min}$ after rapamycin addition. (E) Decay curves plotting percentage of total interactions as a function of distance between interacting points. (F) Same as (D), data from the Brn1 anchor-away strain. (G) Same as (E), but from asynchronous cultures of wild-type and ycs4-2 strains incubated for $2 \mathrm{hr}$ at restrictive temperature. $(\mathrm{H})$ Interaction difference between the wild-type and condensin-depleted cells. Z-scores for all interactions were calculated in both backgrounds. Wild-type was then subtracted from Brn1 depletion. (I) Decay curves for C. elegans nuclei that were spiked into the genome-wide chromosome conformation capture experiments for ycs4-2 shown in (G) and Figure S1B.

Iwasaki et al. 2010). We failed to detect significant association of tRNA genes with the rDNA (Figure S2C), but tRNAtRNA interactions were indeed enriched within the top $1 \%$ of all interactions in wild-type cells. These interactions were predominately interchromosomal (Figure 2D) and reduced upon condensin depletion in our data sets (Figure 2E), 
A

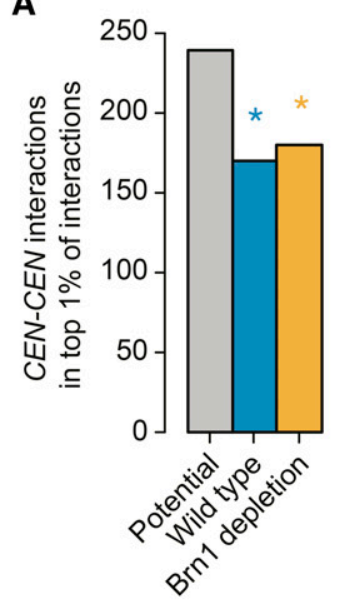

B

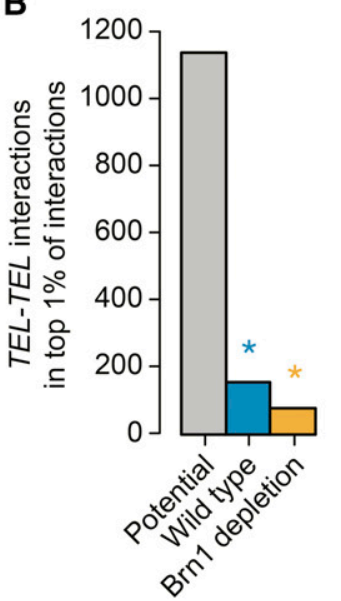

C

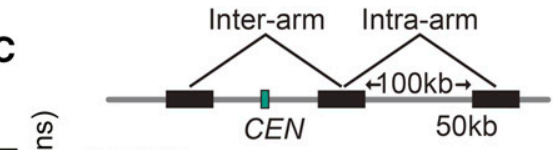

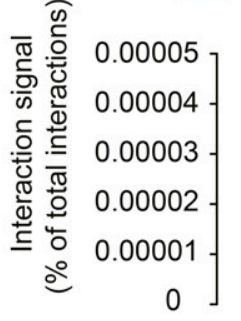

CEN

章
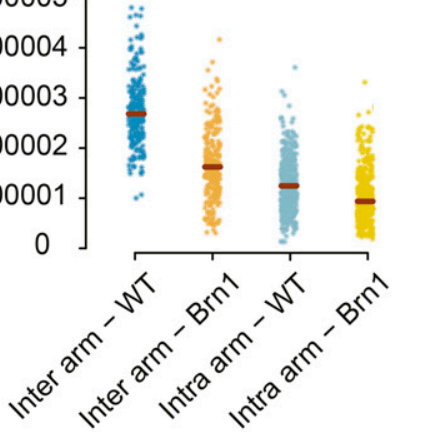

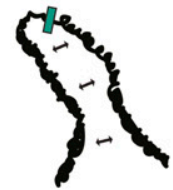

Wild type

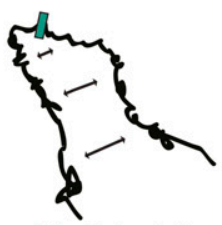

Brn1 depletion

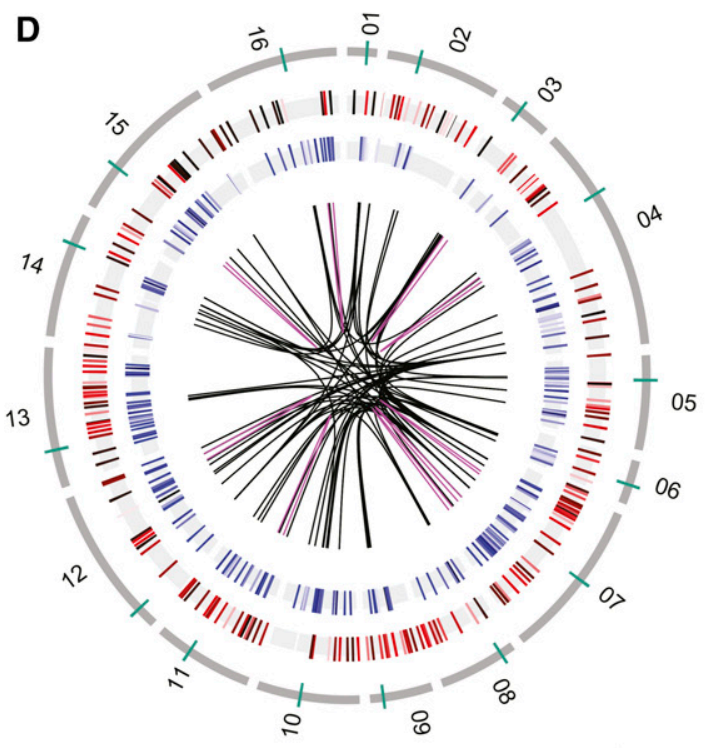

I Centromere

Condensin dependent tRNA interactions:

I Interchromosomal

I Intrachromosomal
F
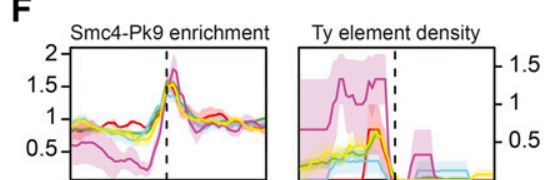

60
-40
-20
0

Pollll subunit enrichment

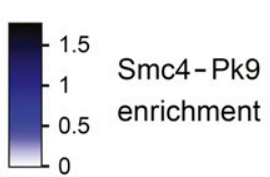

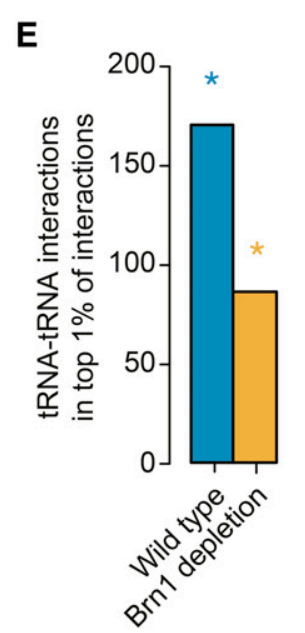
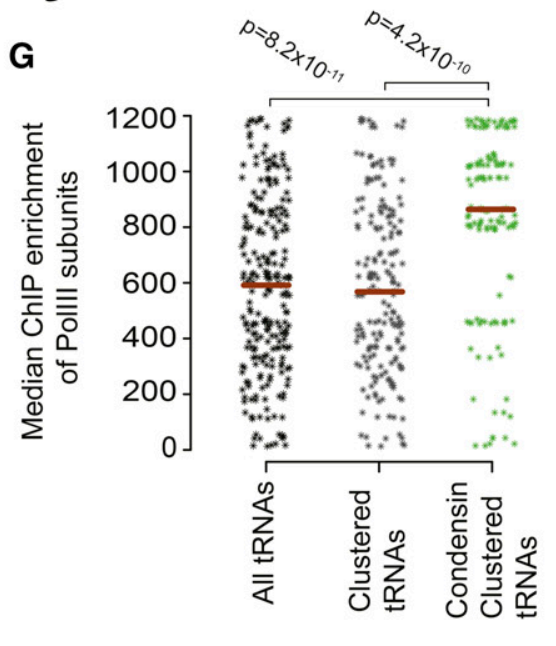
supporting a role for condensin in tRNA gene clustering. Of the 40 tRNA gene families, eight exhibited significant condensin-dependent homotypic interactions (Figure S2D), including the $\mathrm{t}^{\mathrm{LEU}}$ [CAA] genes used in previous FISH experiments (Haeusler et al. 2008). Although tRNA genes are sites of condensin binding (D'Ambrosio et al. 2008; Haeusler et al. 2008; Iwasaki et al. 2010; Kranz et al. 2013), ChIP-sequencing (ChIP-seq) analysis of the condensin subunit Smc4 tagged with $\mathrm{Pk} 9$ revealed no clear correlation between relative condensin enrichment and condensindependent clustering (Figure $2 \mathrm{~F}$ and Figure S2E). This lack of correlation occurs despite the fact that regions of the genome that are enriched for condensin binding show more loss of interactions than unenriched regions (Figure S2F).

To investigate whether condensin-dependent clustering of tRNAs is linked to tRNA gene expression, we analyzed published RNA polymerase (Pol) III binding data (Moqtaderi and Struhl 2004). We observed no direct correlation between the levels of Pol III and condensin at individual tRNA genes. However, tRNA genes that were dependent on condensin for their clustering showed higher Pol III binding compared to other tRNA genes (Figure 2G). It is possible that condensinmediated clustering of these tRNA genes supports higher Pol III binding.

Interestingly, the largest tRNA gene family in the yeast genome ( $\mathrm{t}^{\mathrm{ASP}}[\mathrm{GUC}]$ ) exhibited robust clustering that was unaffected by condensin inactivation (Figure S2D). The reason for this unusual behavior is unclear because the 11 genes of this family are dispersed among other tRNA genes on multiple chromosomes. They are also not enriched near centromeres or telomeres, indicating that the observed condensinindependent clustering is not a result of hitchhiking with those features. To further understand condensin-independent tRNA gene clustering, we analyzed tRNA genes near replication origins whose clustering depends on forkhead transcription factors (Knott et al. 2012). We found that tRNA genes, which are within $1 \mathrm{~kb}$ of replication origins regulated by forkhead transcription factors (accounting for $\sim 5 \%$ of all tRNA genes), do not lose clustering in response to condensin depletion. However, forkhead-mediated tRNA gene clustering does not account for the condensin-independent clustering of the $\mathrm{t}^{\mathrm{ASP}}$ [GUC] family, as only 3 out of the 16 genes are found within $1 \mathrm{~kb}$ of forkhead-regulated origins. We conclude that condensin-mediated clustering occurs for a specific set of tRNA genes and that at least one tRNA gene family uses a condensin-independent clustering mechanism.

\section{Chromosome XII has a unique condensin- dependent organization}

One chief point of condensin binding across the yeast genome is the rDNA (Johzuka et al. 2006; D'Ambrosio et al. 2008; Dulev et al. 2008), which resides in a single $\sim 1$-Mb array in the middle of ChrXII. As observed previously, the rDNA effectively splits ChrXII into two independent interaction domains (Figure 3A) (Duan et al. 2010; Rutledge et al. 2015). Depletion of condensin led to an increase in interactions between the two ChrXII domains (Figure 3B), whereas a strong reduction of local interactions was observed in the genomic regions abutting the rDNA (Figure $3 \mathrm{C}$ ). A similar result was seen in the $y c s 4-2$ mutant (Figure S3, A and B). These data indicate that condensin controls the conformation of the sequences flanking the rDNA array.

Remarkably, the effect of condensin is not symmetric; the centromere-proximal left flank of the rDNA (ChrXII-L) exhibited a stronger and more far-reaching loss of local interactions upon condensin depletion than the centromeredistal right flank (ChrXII-R; Figure S3C) (Lazar-Stefanita et al. 2017). To test if this asymmetry is driven by polar interactions of the rDNA array with either flank, we performed an in silico 4C analysis. We considered the entire rDNA as a single repeat and determined its interactions with the rest of ChrXII (Figure 3D). These analyses revealed that the rDNA interacts more with ChrXII-L than ChrXII-R in wildtype cells, with particularly strong interactions between the rDNA and CEN12 (Figure 3D and Figure S3D). This contact was specific because there was no enrichment of interactions between the rDNA and the centromeres of other chromosomes (Figure S3E). Furthermore, unlike the pairwise interactions between centromeres, the interaction between the rDNA and CEN12 depended on condensin (Figure 3D).

\footnotetext{
taken on every chromosome that was of sufficient size (a total of 17 arms within 10 chromosomes). Interaction scores between these groups are shown for wild-type with rapamycin treatment (WT), or Brn1-FRB with rapamycin treatment (Brn1) (bottom left). A model of a potential mechanism for how condensin complex maintains interarm parity is shown on the right. (D) Circos plot arcs show interactions that are lost from the top $1 \%$ of all interactions upon condensin depletion. These interactions are colored for whether the interaction is interchromosomal (black) or intrachromosomal (pink). Chromatin immunoprecipitation (ChIP) enrichment of SMC4-Pk9 (blue) and RNA polymerase III (Pol III) subunits (red) (Mogtaderi and Struhl 2004) is shown for each tRNA, with the color gradients giving the intensity of binding. (E) Condensin-dependent clustering of tRNAs. On the $y$-axis, the number of homotypic interactions within the top $1 \%$ of all interactions is plotted. Asterisks show significant enrichment of clustering compared to 1000 permutations based on random shuffling of each set of genomic annotation coordinates $(P<0.05)$. (F) (Left panel) Smc4-Pk9 ChIP-seq enrichment scores in the $3 \mathrm{~kb}$ surrounding clustered tRNA genes (those that have interactions in the top $1 \%$ of all interactions). Lowest ChIP scores correspond to the distribution of Ty and LTR elements (right panel), possibly reflecting the low mappability to these repetitive regions. Each tRNA gene was categorized based on whether all of its interactions with other tRNA genes were lost or maintained. Some tRNA genes had a mixture of these categories and/or gained new interactions, which resulted in additional mixed categories. No tRNA genes only gained interactions, so this category is omitted. (G) Median ChIP enrichment of several Pol III subunits (Moqtaderi and Struhl 2004) at all tRNAs, tRNAs that cluster together (in the top 1\% of all interactions), and tRNAs that lose clustering after condensin depletion. Significance test between groups was based on a Mann-Whitney $U$ test. Group medians are demarcated with the horizontal bar.
} 

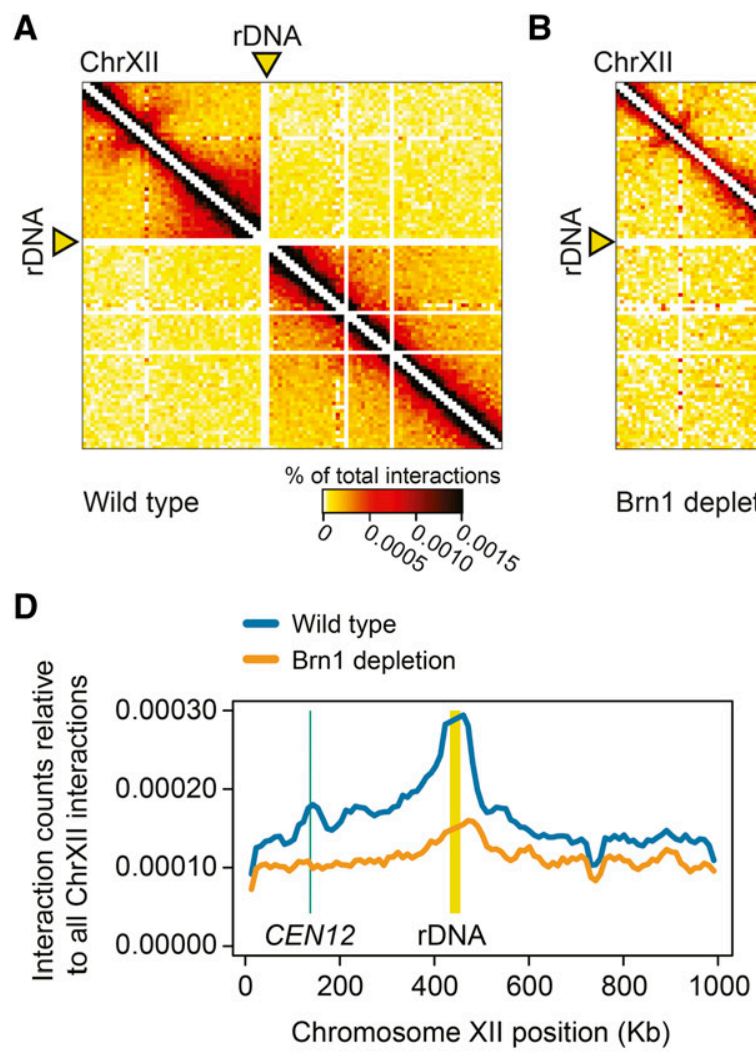

\section{$\mathbf{F}$}

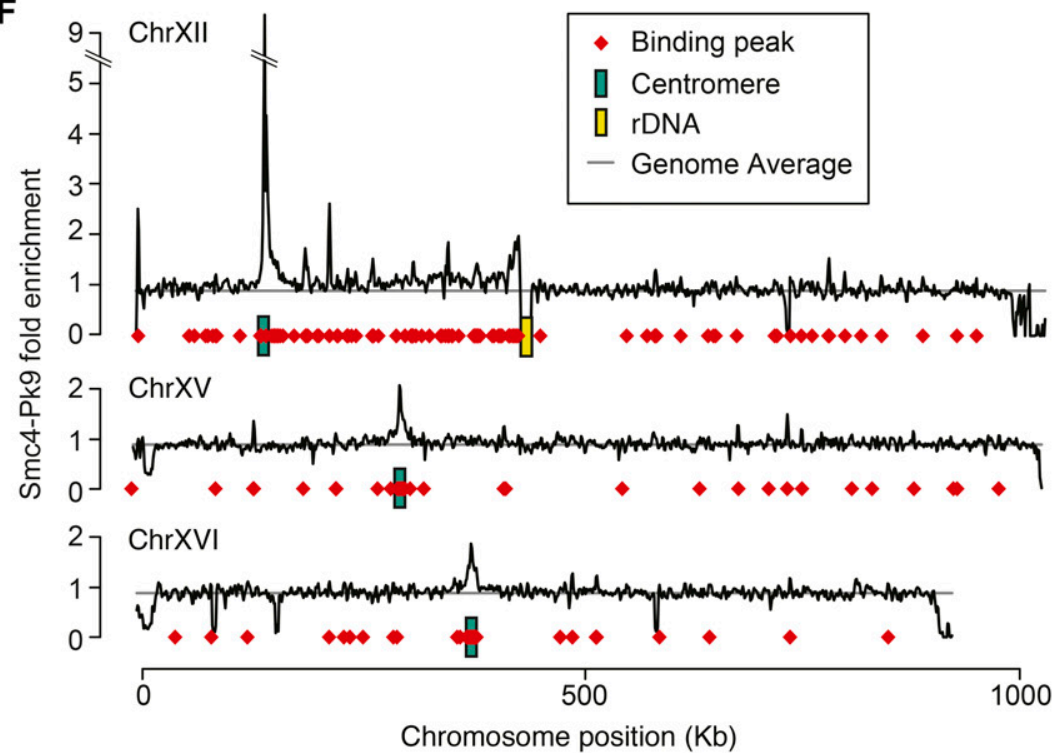

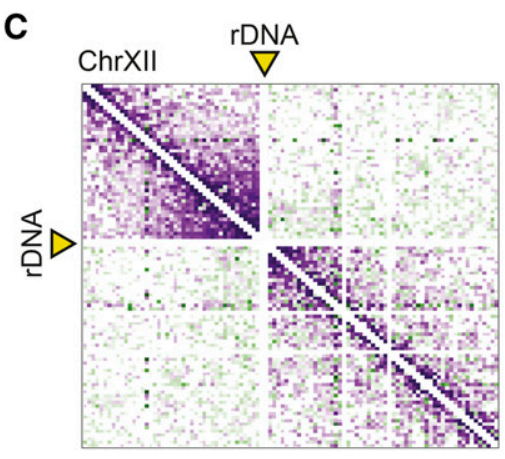

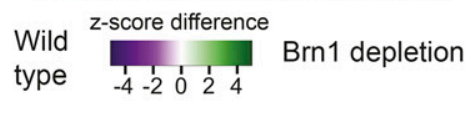

E

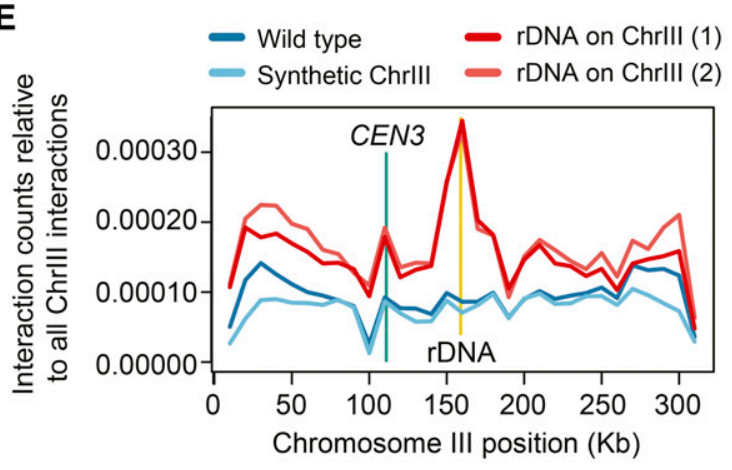

G Smc4-Pk9 $\quad 4 \quad 3 \quad 2 \quad 1 \quad 0$ fold enrichment

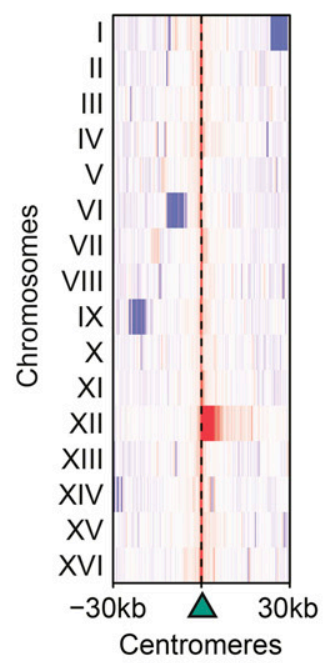

Figure 3 Condensin-mediated organization of the rDNA-containing chromosome (Chr) XII. ChrXII interaction map from asynchronously growing yeast cells without (A) and with (B) condensin depletion using anchor-away. (C) Z-score subtraction of wild-type from condensin depletion data shown in (A and B). (D) In silico 4C analysis using the rDNA as bait to assess its interaction profile with the rest of ChrXII. (E) In silico 4C of rDNA using previously published Hi-C data (Mercy et al. 2017). The red lines show profiles from two independently constructed strains in which the rDNA array was inserted into the middle of a synthetic Chrlll. The blue lines show profiles of wild-type and synthetic Chrlll. (F) Smc4-Pk9 chromatin immunoprecipitation (ChIP) enrichment along three similarly sized chromosomes in asynchronous cells. Data were smoothed using 30-kb windows sliding over 10-kb steps. Red diamonds: condensin binding peaks from MACS2 analysis; green boxes: centromeres; and yellow box: rDNA. The gray line indicates genome average. (G) ChIP-sequencing enrichment of Smc4-Pk9 in 50-bp windows across the $60 \mathrm{~kb}$ surrounding each centromere. 
The interaction between the rDNA and CEN12 could be the result of specialized tethering sequences near CEN12 or of their presence on the same chromosome. To distinguish between these possibilities, we used previously published Hi-C data from a synthetic yeast strain in which the rDNA array was inserted into ChrIII (Mercy et al. 2017). Transposition of the rDNA into ChrIII was sufficient to create two domains on ChrIII, similar to what is observed for the native ChrXII (Duan et al. 2010; Rutledge et al. 2015; Mercy et al. 2017). Moreover, in-silico 4C analysis of this strain showed interaction between the rDNA and CEN3, whereas the interaction with CEN12 was lost (Figure 3E and Figure S3F), suggesting that the rDNA specifically interacts with the centromere of the same chromosome. It is possible that condensin-mediated tethering of rDNA to the centromere [reported to occur specifically during anaphase (Lazar-Stefanita et al. 2017)] may be important for proper segregation of the rDNA array, for which condensin function was shown to be essential (Freeman et al. 2000; Lavoie et al. 2004).

The condensin dependence of the rDNA-CEN12 interaction suggested that condensin might be particularly active in the interval between these two loci. Indeed, analysis of Smc4-Pk9 binding along ChrXII revealed a specific enrichment of condensin between the rDNA and CEN12 in wild-type cells (Figure 3F). Condensin binding is sharply delimited, as binding levels are at the genome-wide average on the left side of CEN12 (Figure 3F). No other centromere showed a comparably asymmetric condensin enrichment (Figure $3 \mathrm{G})$. These data indicate that the genomic interval between CEN12 and the rDNA forms a domain of increased condensin binding and condensin-dependent interactions akin to TADs in higher eukaryotes.

\section{Condensin-dependent changes to chromosomal interactions are not coupled to global changes in gene expression}

Given the broad effects of condensin on chromosome compaction, we sought to define the effect of these changes on gene expression. To quantify global changes in gene expression levels, we performed spike-in normalized mRNA-seq analysis upon condensin inactivation. Unexpectedly, there were no gross changes to global levels of gene expression following acute condensin depletion by anchor-away (Figure 4A). Although it is possible that $30 \mathrm{~min}$ of condensin depletion are not sufficient to mount a robust transcriptional response, we do not favor this idea because transcriptional responses in yeast occur within minutes (Miller et al. 2011; Mouaikel et al. 2013) and this time frame is sufficient to observe a robust loss of compaction by $3 \mathrm{C}$-seq. Thus, the changes in chromosomal interactions observed upon anchor-away depletion of condensin do not lead to immediate changes in global transcription.

To further test this apparent resilience of the transcriptional program to genome decompaction, we also analyzed global expression levels in $y c s 4-2$ and ycg1-2 mutants after $2 \mathrm{hr}$ of incubation at restrictive temperature (Figure 4, B and
C). The $y c g$ 1-2 harbors five nonsynonymous mutations to the coding sequence (Figure S4A). Interestingly, the ycs4-2 and $y c g$ 1-2 mutations were associated with a temperatureindependent effect on the global level of gene expression. Both at the permissive and restrictive temperatures, the $y \operatorname{cs} 4-2$ and ycg1-2 strains exhibited slightly increased average expression compared to wild-type (Figure 4, B and C). These data suggest that the $y c s 4-2$ and $y c g 1-2$ mutations are not fully functional at the permissive temperature, consistent with previous reports (Haeusler et al. 2008; Wang et al. 2016).

To further separate the effects of temperature and the ycs4-2 mutation, we hierarchically clustered genes across the different conditions based on expression level. This analysis revealed four major patterns of gene expression (Figure 4D). Cluster 1 represents a substantial proportion of the genome and was sensitive to the ycs4-2 mutation. Clusters 2 and 3 reflected temperature-dependent changes to gene expression with gene expression increasing or decreasing, respectively. Lastly, cluster 4 included genes that responded to temperature in wild-type but not upon ycs 4-2 mutation. Importantly, gene ontology analysis did not find enrichment of cell cycle-controlled genes in any of the clusters, indicating that the expression changes were not secondary to a cell cycle arrest at the restrictive temperature. Overall, almost $50 \%$ of the genome displayed increased expression in the ycs4-2 strain compared to wild-type at $23^{\circ}$, the permissive temperature. Finally, we also evaluated if the four gene expression clusters defined by the ycs4-2 data capture any condensindependent changes in the anchor-away data. There was no difference in the expression of the four clusters upon condensin depletion by Brn1 anchor-away (Figure S4B).

Notably, the biggest effect of $y c s 4-2$ on gene expression was observed on ChrXII, with a particular enrichment of upregulated genes on the right side of ChrXII (ChrXII-R) (Figure 4E). This effect was not observed in anchor-away or ycg1-2 strains (Figure 4F), and was not due to changes in copy number (Figure S4C) or differences in the size of the rDNA array (Figure S4D). Interestingly, the YCS4 gene is located on ChrXII-R, within the region containing an enrichment of genes that were upregulated in the ycs4-2 strain, and mRNA levels of the ycs4-2 allele were elevated approximately twofold compared to wild-type. Thus, the ycs 4-2 strain may have adapted transcriptionally to compensate for the effect of the condensin mutation.

Previous studies have suggested that condensin mediates silencing of Pol II-transcribed genes adjacent to tRNA genes on plasmid constructs (Haeusler et al. 2008). Analyzing the effect of condensin depletion and mutations on the expression of genes close to tRNAs did not support a role for condensinmediated repression adjacent to tRNA genes (Figure S4E). We also did not observe a direct correlation between condensin binding and gene repression. Compared to the rest of the genome, the central portion of ChrXII between the centromere and rDNA (ChrXII-L2) is more enriched for condensin (Figure 3F) and loses more chromosomal interactions upon condensin depletion (Figure 3D). Yet, this region did not 

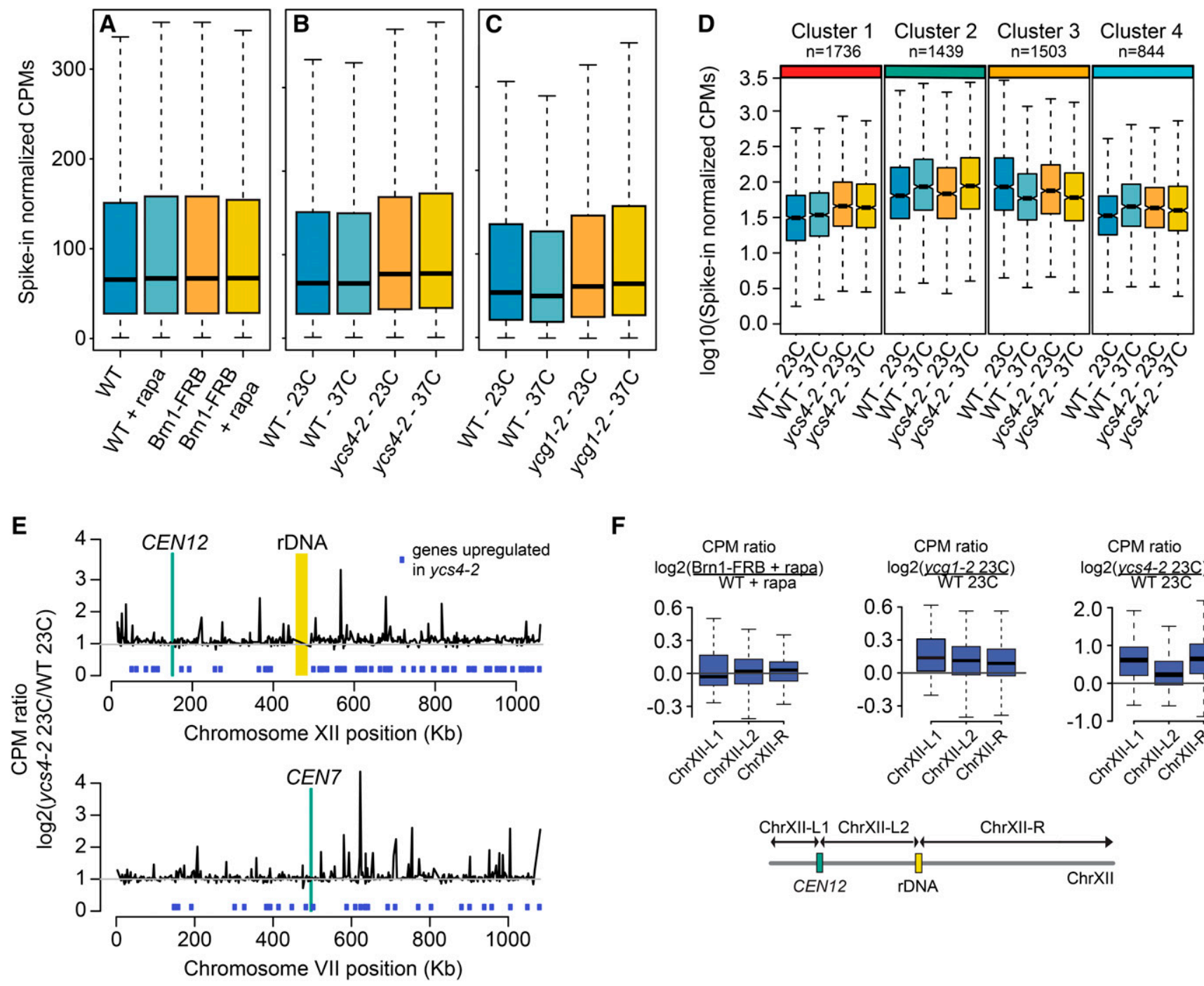

$\mathbf{F}$
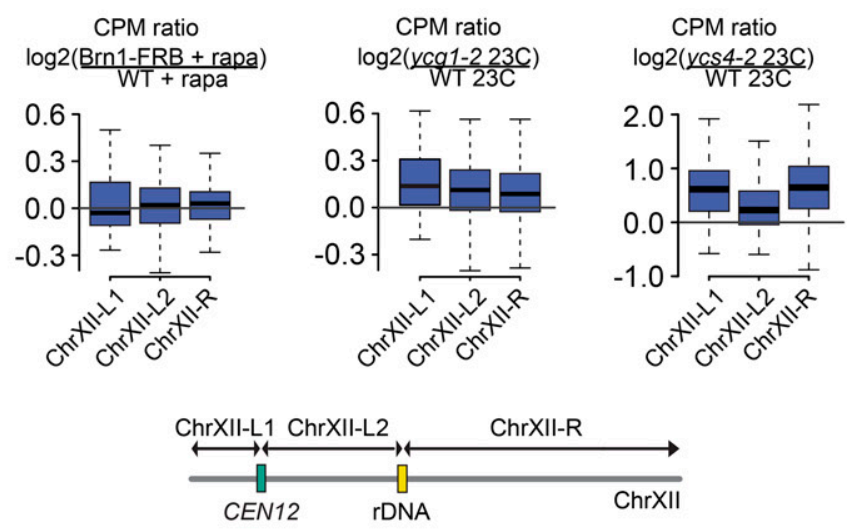

Figure 4 mRNA-sequencing analysis following condensin perturbation. (A-C) Spike-in corrected counts per million (CPM) for all genes are plotted. (A) Data are from wild-type (WT) parental and Brn1 anchor-away strains grown asynchronously and collected with or without rapamycin addition for 30 min. (B) WT and $y c s 4-2$ strain incubated at permissive $\left(23^{\circ}\right)$ or restrictive $\left(37^{\circ}\right)$ temperature for $2 \mathrm{hr}$. (C) WT and ycg1-2 strain incubated at permissive $\left(23^{\circ}\right)$ or restrictive $\left(37^{\circ}\right)$ temperature for $2 \mathrm{hr}$. (D) Four gene groups were generated by hierarchical clustering of gene expression from ycs4-2 and WT strains grown at permissive and restrictive temperature. Expression levels of genes within each cluster are shown for each growth condition. (E) Average gene expression ratios [from (B)] within $30-\mathrm{kb}$ windows sliding with a 10-kb step across chromosomes (Chrs) XII (top) and VII (bottom). Differentially expressed genes were determined by edgeR and labeled with blue squares. (F) Gene expression ratios plotted for three different regions of ChrXII (bottom schematic). Expression ratio is between Brn1 anchor

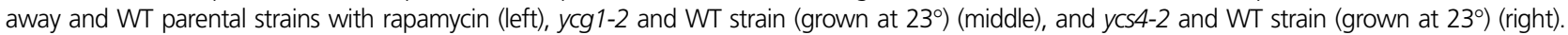

show any specific upregulation upon condensin depletion or mutations (Figure 4E). We conclude that genome decompaction triggered by removal of condensin does not have immediate effects on global gene expression.

\section{Discussion}

Here, we show that condensin is essential for the organization of the whole $S$. cerevisiae genome without controlling global gene expression levels. In asynchronous cells, the primary effect of condensin depletion and mutation was a reduction in the frequency of short-range interactions, suggesting that yeast condensin promotes local interactions $(<100 \mathrm{~kb})$.
Condensin-mediated interactions at this scale are consistent with the increase of local intrachromosomal interactions in mitotic chromosomes of $S$. cerevisiae (Lazar-Stefanita et al. 2017), and computational models of chromosome compaction before and during early prophase in humans (Naumova et al. 2013; Goloborodko et al. 2016a).

The short-range interactions mediated by condensin in S. cerevisiae differ from observations in other organisms, likely reflecting differences in genome organization. Whereas in S. cerevisiae, chromosome interactions $<100 \mathrm{~kb}$ are increased during mitosis, in $S$. pombe, chromosomal interactions between $\sim 100 \mathrm{~kb}$ and $1 \mathrm{Mb}$ are increased at the expense of interactions $<100 \mathrm{~kb}$ and $>1 \mathrm{Mb}$ (Kim et al. 2016; Kakui 
et al. 2017). It is possible that $S$. cerevisiae and $S$. pombe condensins have diverged to mediate different ranges of interactions based on chromosome length, as $S$. cerevisiae chromosomes range between 0.2 and $1.5 \mathrm{Mb}$, while $S$. pombe chromosomes are $\sim 2.5-5.5 \mathrm{Mb}$ in length. Different ranges of activity have also been proposed for the two types of condensins in metazoans. Recent studies in chicken (Gibcus et al. 2017) and human (Walther et al. 2018) cells found that condensin II mediates the formation of progressively longer loops of chromatin, whereas condensin I produces short loops, nested within the longer ones. Thus, condensins in different species, as well as different condensin types within a single species, likely evolved to carry out different sizerange interactions.

The molecular mechanism underlying the size of condensinmediated interactions may be based on the "loop extrusion" model, which has been gaining recent traction (Nasmyth 2001; Alipour and Marko 2012; Goloborodko et al. 2016b). In this model, a DNA loop gets progressively larger as it is pushed through the condensin ring. If condensin were repeatedly unloaded from chromosomes due to instability and/or the processivity of DNA extrusion was slow, the result would be condensins mediating short-range interactions (Gerlich et al. 2006; Goloborodko et al. 2016a). Conversely, stable association of condensin (or specific condensin types) may lead to increased loop lengths and longer-range interactions, which have been seen in experiments analyzing mitotic chromosome structure (Gibcus et al. 2018; Walther et al. 2018). Uncovering the molecular mechanisms that regulate loop extrusion will be important to for our understanding of the dynamic changes that are mediated by condensins during the cell cycle.

In addition to a genome-wide effect on short-range interactions, our analyses show that yeast condensin promotes specific long-range and interchromosomal interactions between tRNA genes (Figure 2D). While loop extrusion is an effective model to explain the formation of chromosomal loops at different scales, how condensin mediates interchromosomal interactions, such as those between certain tRNA genes, remains unclear. In vitro, yeast condensin is capable of mediating trans interactions between two DNA molecules (Terakawa et al. 2017). Therefore, it is possible that condensins directly create interchromosomal links between tRNA genes by encircling DNA or indirectly by interacting with specific recruiting proteins (Figure S4F).

The notion that condensin functions at specific loci to promote interchromosomal interactions is also supported by analyses in fission yeast, where condensin was shown to mediate clustering of cell cycle-regulated and tRNA genes (Kim et al. 2016). In $S$. pombe, condensin represses tRNA transcription (Iwasaki et al. 2010). In S. cerevisiae, our results indicate that tRNA genes clustered by condensin have higher levels of RNA Pol III (Figure 2G). How these data fit together is unclear, but it is likely that the function of condensinmediated tRNA clustering is to support tighter regulation of tRNA expression.
One possible consequence of condensin-mediated chromosomal interactions is the formation of defined TAD structures, as observed in fission yeast (Kim et al. 2016), D. melanogaster (Van Bortle et al. 2014), and in C. elegans (Crane et al. 2015). Our data support a similar function in budding yeast, albeit at a more limited scale. In particular, condensin is required for a nested condensin-enriched domain between the rDNA and CEN12. Similar to TAD boundaries (Rowley and Corces 2016), both domain boundaries, the rDNA and CEN12, are highly bound by condensin. It is possible that when strong condensin recruitment domains, such as the rDNA and CEN12, are located adjacent to each other, condensin binding is enhanced in the intervening region. Such behavior would be akin to C. elegans dosage compensation, where a specialized condensin complex is recruited to specific recruitment sites, from which it spreads out (Albritton et al. 2017).

In C. elegans and other organisms, condensins have been linked to the regulation of transcription (Iwasaki et al. 2010; Lau and Csankovszki 2014; Wang et al. 2017; Swygert et al. 2018). Our data indicate that the global gene expression program is largely unaffected by condensin depletion in cycling yeast. We did observe a slight genome-wide increase in gene expression in ycs4-2 and ycg1-2 mutants. As the $y c s 4-2$ and $y c g 1-2$ mutations already cause transcriptional phenotypes at room temperature (Haeusler et al. 2008; Wang et al. 2016), these differences could be an indirect result of the chronic presence of the condensin mutations as opposed to the acute response to rapid depletion of condensin from the nucleus by the anchor-away system. A recent preprint reported that in $S$. cerevisiae condensin is required for the compaction and repression of gene expression during quiescence (Swygert et al. 2018). This may reflect quiescence-specific regulation of the condensin complex, or more chronic effects of condensin depletion as in ycs4-2 and $y c g 1-2$, because condensin was depleted from quiescent cells for over a week.

The observation that acute depletion of condensin caused immediate effects on genome organization but not on gene expression suggests that transcription can be decoupled from global chromosome structure. However, we note that chromosome condensation in $S$. cerevisiae is substantially less dramatic than observed for mitotic chromosomes in larger eukaryotes (Gottschling and Berg 1998). Furthermore, gene regulatory elements in yeast are localized near the promoters, and may not depend on specific chromosomal interactions as enhancers and promoters in bigger eukaryotic genomes. Finally, other energy-dependent chromatin organizers, including other SMC proteins, may compensate for the loss of condensin and provide robustness against acute changes in condensin activity.

\section{Acknowledgments}

We thank H. Yu for sharing strains, M. Imakaev for help establishing the analysis pipeline, D. Corrales and H. He for 
help with $S$. pombe culture and primers, and the New York University Department of Biology Sequencing Core for technical assistance and data processing. This work was supported by a fellowship from Boehringer Ingelheim Fonds to M.R.P., and National Institutes of Health grants R01 GM-111715 to A.H. and R01 GM-107293 to S.E.

\section{Literature Cited}

Albritton, S. E., A. L. Kranz, L. H. Winterkorn, L. A. Street, and S. Ercan, 2017 Cooperation between a hierarchical set of recruitment sites targets the $\mathrm{X}$ chromosome for dosage compensation. Elife 6: e23645. https://doi.org/10.7554/eLife.23645

Alipour, E., and J. F. Marko, 2012 Self-organization of domain structures by DNA-loop-extruding enzymes. Nucleic Acids Res. 40: 11202-11212. https://doi.org/10.1093/nar/gks925

Anders, S., P. T. Pyl, and W. Huber, 2015 HTSeq-a python framework to work with high-throughput sequencing data. Bioinformatics 31: 166-169. https://doi.org/10.1093/bioinformatics/btu638

Blitzblau, H. G., C. S. Chan, A. Hochwagen, and S. P. Bell, 2012 Separation of DNA replication from the assembly of break-competent meiotic chromosomes. PLoS Genet. 8: e1002643. https://doi.org/10.1371/journal.pgen.1002643

Crane, E., Q. Bian, R. P. McCord, B. R. Lajoie, B. S. Wheeler et al., 2015 Condensin-driven remodelling of X chromosome topology during dosage compensation. Nature 523: 240-244. https://doi.org/10.1038/nature14450

Cuylen, S., J. Metz, A. Hruby, and C. H. Haering, 2013 Entrapment of chromosomes by condensin rings prevents their breakage during cytokinesis. Dev. Cell 27: 469-478. https://doi.org/ 10.1016/j.devcel.2013.10.018

D'Ambrosio, C., C. K. Schmidt, Y. Katou, G. Kelly, T. Itoh et al., 2008 Identification of cis-acting sites for condensin loading onto budding yeast chromosomes. Genes Dev. 22: 2215-2227. https://doi.org/10.1101/gad.1675708

Dekker, J., and L. Mirny, 2016 The 3D genome as moderator of chromosomal communication. Cell 164: 1110-1121. https:// doi.org/10.1016/j.cell.2016.02.007

Dixon, J. R., S. Selvaraj, F. Yue, A. Kim, Y. Li et al., 2012 Topological domains in mammalian genomes identified by analysis of chromatin interactions. Nature 485: 376-380. https://doi.org/ 10.1038 /nature 11082

Dixon, J. R., I. Jung, S. Selvaraj, Y. Shen, J. E. Antosiewicz-Bourget et al., 2015 Chromatin architecture reorganization during stem cell differentiation. Nature 518: 331-336. https://doi. org/10.1038/nature14222

Dixon, J. R., D. U. Gorkin, and B. Ren, 2016 Chromatin domains: the unit of chromosome organization. Mol. Cell 62: 668-680. https://doi.org/10.1016/j.molcel.2016.05.018

Duan, Z., M. Andronescu, K. Schutz, S. McIlwain, Y. J. Kim et al., 2010 A three-dimensional model of the yeast genome. Nature 465: 363-367. https://doi.org/10.1038/nature08973

Dulev, S., L. Aragon, and A. Strunnikov, 2008 Unreplicated DNA in mitosis precludes condensin binding and chromosome condensation in S. cerevisiae. Front. Biosci. 13: 5838-5846. https:// doi.org/10.2741/3120

Edstrom, J. E., W. Grampp, and N. Schor, 1961 The intracellular distribution and heterogeneity of ribonucleic acid in starfish oocytes. J. Biophys. Biochem. Cytol. 11: 549-557. https://doi. org/10.1083/jcb.11.3.549

Freeman, L., L. Aragon-Alcaide, and A. Strunnikov, 2000 The condensin complex governs chromosome condensation and mitotic transmission of rDNA. J. Cell Biol. 149: 811-824. https://doi. org/10.1083/jcb.149.4.811
Frenster, J. H., V. G. Allfrey, and A. E. Mirsky, 1963 Repressed and active chromatin isolated from interphase lymphocytes. Proc. Natl. Acad. Sci. USA 50: 1026-1032. https://doi.org/10.1073/ pnas.50.6.1026

Gerlich, D., T. Hirota, B. Koch, J. M. Peters, and J. Ellenberg, 2006 Condensin I stabilizes chromosomes mechanically through a dynamic interaction in live cells. Curr. Biol. 16: 333-344. https://doi.org/10.1016/j.cub.2005.12.040

Gibcus, J. H., K. Samejima, A. Goloborodko, I. Samejima, N. Naumova et al., 2017 Mitotic chromosomes fold by condensin-dependent helical winding of chromatin loop arrays. bioRxiv. Available at: https://doi.org/10.1101/174649.

Gibcus, J. H., K. Samejima, A. Goloborodko, I. Samejima, N. Naumova et al., 2018 A pathway for mitotic chromosome formation. Science 359: eaao6135. https://doi.org/10.1126/science.aao6135

Giorgio, E., D. Robyr, M. Spielmann, E. Ferrero, E. Di Gregorio et al., 2015 A large genomic deletion leads to enhancer adoption by the lamin B1 gene: a second path to autosomal dominant adult-onset demyelinating leukodystrophy (ADLD). Hum. Mol. Genet. 24: 3143-3154. https://doi.org/10.1093/ hmg/ddv065

Gligoris, T., and J. Lowe, 2016 Structural insights into ring formation of cohesin and related Smc complexes. Trends Cell Biol. 26: 680-693. https://doi.org/10.1016/j.tcb.2016.04.002

Goloborodko, A., M. V. Imakaev, J. F. Marko, and L. Mirny, 2016a Compaction and segregation of sister chromatids via active loop extrusion. Elife 5: e14864. https://doi.org/10.7554/ eLife.14864

Goloborodko, A., J. F. Marko, and L. A. Mirny, 2016b Chromosome compaction by active loop extrusion. Biophys. J. 110: 2162-2168. https://doi.org/10.1016/j.bpj.2016.02.041

Gottschling, D. E., and B. L. Berg, 1998 Chromosome dynamics: yeast pulls it apart. Curr. Biol. 8: R76-R79. https://doi.org/ 10.1016/S0960-9822(98)70050-8

Gottschling, D. E., O. M. Aparicio, B. L. Billington, and V. A. Zakian, 1990 Position effect at S. cerevisiae telomeres: reversible repression of Pol II transcription. Cell 63: 751-762. https://doi. org/10.1016/0092-8674(90)90141-Z

Haeusler, R. A., M. Pratt-Hyatt, P. D. Good, T. A. Gipson, and D. R. Engelke, 2008 Clustering of yeast tRNA genes is mediated by specific association of condensin with tRNA gene transcription complexes. Genes Dev. 22: 2204-2214. https://doi.org/10.1101/ gad.1675908

Hartl, T. A., H. F. Smith, and G. Bosco, 2008 Chromosome alignment and transvection are antagonized by condensin II. Science 322: 1384-1387. https://doi.org/10.1126/science.1164216

Haruki, H., J. Nishikawa, and U. K. Laemmli, 2008 The anchoraway technique: rapid, conditional establishment of yeast mutant phenotypes. Mol. Cell 31: 925-932. https://doi.org/ 10.1016/j.molcel.2008.07.020

Heitz, E., 1928 Das heterochromatin der moose. Jahrb. Wiss. Botanik. 69: 762-818.

Hirano, T., 2016 Condensin-based chromosome organization from bacteria to vertebrates. Cell 164: 847-857. https://doi.org/ 10.1016/j.cell.2016.01.033

Hsieh, T.-H., G. Fudenberg, A. Goloborodko, and O. Rando, 2016 Micro-C XL: assaying chromosome conformation at length scales from the nucleosome to the entire genome. Nat. Methods 13: 1009-1011.

Ibn-Salem, J., S. Kohler, M. I. Love, H. R. Chung, N. Huang et al., 2014 Deletions of chromosomal regulatory boundaries are associated with congenital disease. Genome Biol. 15: 423. https:// doi.org/10.1186/s13059-014-0423-1

Imakaev, M., G. Fudenberg, R. P. McCord, N. Naumova, A. Goloborodko et al., 2012 Iterative correction of Hi-C data reveals hallmarks of chromosome organization. Nat. Methods 9: 999-1003. https:// doi.org/10.1038/nmeth. 2148 
Iwasaki, O., A. Tanaka, H. Tanizawa, S. I. Grewal, and K. Noma, 2010 Centromeric localization of dispersed Pol III genes in fission yeast. Mol. Biol. Cell 21: 254-265. https://doi.org/10.1091/ mbc.e09-09-0790

Ji, X., D. B. Dadon, B. E. Powell, Z. P. Fan, D. Borges-Rivera et al., 2016 3D chromosome regulatory landscape of human pluripotent cells. Cell Stem Cell 18: 262-275. https://doi.org/10.1016/ j.stem.2015.11.007

Jin, F., Y. Li, J. R. Dixon, S. Selvaraj, Z. Ye et al., 2013 A highresolution map of the three-dimensional chromatin interactome in human cells. Nature 503: 290-294. https://doi.org/10.1038/ nature 12644

Jin, Q. W., J. Fuchs, and J. Loidl, 2000 Centromere clustering is a major determinant of yeast interphase nuclear organization. J. Cell Sci. 113: 1903-1912.

Johzuka, K., M. Terasawa, H. Ogawa, T. Ogawa, and T. Horiuchi, 2006 Condensin loaded onto the replication fork barrier site in the rRNA gene repeats during $S$ phase in a FOB1-dependent fashion to prevent contraction of a long repetitive array in Saccharomyces cerevisiae. Mol. Cell. Biol. 26: 2226-2236. https:// doi.org/10.1128/MCB.26.6.2226-2236.2006

Kakui, Y., A. Rabinowitz, D. J. Barry, and F. Uhlmann, 2017 Condensin-mediated remodeling of the mitotic chromatin landscape in fission yeast. Nat. Genet. 49: 1553-1557. https://doi.org/10.1038/ng.3938

Kim, K. D., H. Tanizawa, O. Iwasaki, and K. Noma, 2016 Transcription factors mediate condensin recruitment and global chromosomal organization in fission yeast. Nat. Genet. 48: 1242-1252. https:// doi.org/10.1038/ng.3647

Kinoshita, K., and T. Hirano, 2017 Dynamic organization of mitotic chromosomes. Curr. Opin. Cell Biol. 46: 46-53. https://doi. org/10.1016/j.ceb.2017.01.006

Knott, S. R., J. M. Peace, A. Z. Ostrow, Y. Gan, A. E. Rex et al., 2012 Forkhead transcription factors establish origin timing and long-range clustering in S. cerevisiae. Cell 148: 99-111. https://doi.org/10.1016/j.cell.2011.12.012

Kramer, M., A. L. Kranz, A. Su, L. H. Winterkorn, S. E. Albritton et al., 2015 Developmental dynamics of X-chromosome dosage compensation by the DCC and H4K20me1 in C. elegans. PLoS Genet. 11: e1005698 [corrigenda: PLoS Genet. 12: e1005899 (2016)]. https://doi.org/10.1371/journal.pgen.1005698

Kranz, A. L., C. Y. Jiao, L. H. Winterkorn, S. E. Albritton, M. Kramer et al., 2013 Genome-wide analysis of condensin binding in Caenorhabditis elegans. Genome Biol. 14: R112. https://doi. org/10.1186/gb-2013-14-10-r112

Kschonsak, M., and C. H. Haering, 2015 Shaping mitotic chromosomes: from classical concepts to molecular mechanisms. BioEssays 37: 755-766. https://doi.org/10.1002/bies.201500020

Langmead, B., C. Trapnell, M. Pop, and S. L. Salzberg, 2009 Ultrafast and memory-efficient alignment of short DNA sequences to the human genome. Genome Biol. 10: R25. https://doi.org/10.1186/ gb-2009-10-3-r25

Lau, A. C., and G. Csankovszki, 2014 Condensin-mediated chromosome organization and gene regulation. Front. Genet. 5: 473. https://doi.org/10.3389/fgene.2014.00473

Lavoie, B. D., E. Hogan, and D. Koshland, 2002 In vivo dissection of the chromosome condensation machinery: reversibility of condensation distinguishes contributions of condensin and cohesin. J. Cell Biol. 156: 805-815. https://doi.org/10.1083/jcb.200109056

Lavoie, B. D., E. Hogan, and D. Koshland, 2004 In vivo requirements for rDNA chromosome condensation reveal two cell-cycleregulated pathways for mitotic chromosome folding. Genes Dev. 18: 76-87. https://doi.org/10.1101/gad.1150404

Lazar-Stefanita, L., V. F. Scolari, G. Mercy, H. Muller, T. M. Guerin et al., 2017 Cohesins and condensins orchestrate the 4D dynamics of yeast chromosomes during the cell cycle. EMBO J. 36: 2684-2697. https://doi.org/10.15252/embj.201797342
Leonard, J., N. Sen, R. Torres, T. Sutani, A. Jarmuz et al., 2015 Condensin relocalization from centromeres to chromosome arms promotes Top2 recruitment during anaphase. Cell Rep. 13: 2336-2344. https://doi.org/10.1016/j.celrep.2015.11.041

Lettice, L. A., S. Daniels, E. Sweeney, S. Venkataraman, P. S. Devenney et al., 2011 Enhancer-adoption as a mechanism of human developmental disease. Hum. Mutat. 32: 1492-1499. https:// doi.org/10.1002/humu.21615

Li, L., X. Lyu, C. Hou, N. Takenaka, H. Q. Nguyen et al., 2015 Widespread rearrangement of 3D chromatin organization underlies polycomb-mediated stress-induced silencing. Mol. Cell 58: 216-231. https://doi.org/10.1016/j.molcel.2015.02.023

Lieberman-Aiden, E., N. L. van Berkum, L. Williams, M. Imakaev, T. Ragoczy et al., 2009 Comprehensive mapping of longrange interactions reveals folding principles of the human genome. Science 326: 289-293. https://doi.org/10.1126/science. 1181369

Lupiáñez, D. G., K. Kraft, V. Heinrich, P. Krawitz, F. Brancati et al., 2015 Disruptions of topological chromatin domains cause pathogenic rewiring of gene-enhancer interactions. Cell 161: 1012-1025. https://doi.org/10.1016/j.cell.2015.04.004

Mercy, G., J. Mozziconacci, V. F. Scolari, K. Yang, G. Zhao et al., 2017 3D organization of synthetic and scrambled chromosomes. Science 355: eaaf4597. https://doi.org/10.1126/science.aaf4597

Miller, C., B. Schwalb, K. Maier, D. Schulz, S. Dumcke et al., 2011 Dynamic transcriptome analysis measures rates of mRNA synthesis and decay in yeast. Mol. Syst. Biol. 7: 458. https://doi. org/10.1038/msb.2010.112

Moqtaderi, Z., and K. Struhl, 2004 Genome-wide occupancy profile of the RNA polymerase III machinery in Saccharomyces cerevisiae reveals loci with incomplete transcription complexes. Mol. Cell. Biol. 24: 4118-4127. https://doi.org/10.1128/ MCB.24.10.4118-4127.2004

Mouaikel, J., S. Z. Causse, M. Rougemaille, Y. Daubenton-Carafa, C. Blugeon et al., 2013 High-frequency promoter firing links THO complex function to heavy chromatin formation. Cell Rep. 5: 1082-1094. https://doi.org/10.1016/j.celrep.2013.10.013

Nakazawa, N., K. Sajiki, X. Xu, A. Villar-Briones, O. Arakawa et al., 2015 RNA pol II transcript abundance controls condensin accumulation at mitotically up-regulated and heat-shock-inducible genes in fission yeast. Genes Cells 20: 481-499. https://doi.org/ 10.1111/gtc. 12239

Nasmyth, K., 2001 Disseminating the genome: joining, resolving, and separating sister chromatids during mitosis and meiosis. Annu. Rev. Genet. 35: 673-745. https://doi.org/10.1146/ annurev.genet.35.102401.091334

Naumova, N., M. Imakaev, G. Fudenberg, Y. Zhan, B. R. Lajoie et al., 2013 Organization of the mitotic chromosome. Science 342: 948-953. https://doi.org/10.1126/science.1236083

Nishide, K., and T. Hirano, 2014 Overlapping and non-overlapping functions of condensins I and II in neural stem cell divisions. PLoS Genet. 10: e1004847. https://doi.org/10.1371/journal. pgen.1004847

Palladino, F., T. Laroche, E. Gilson, A. Axelrod, L. Pillus et al., 1993 SIR3 and SIR4 proteins are required for the positioning and integrity of yeast telomeres. Cell 75: 543-555. https://doi. org/10.1016/0092-8674(93)90388-7

Parkhomchuk, D., T. Borodina, V. Amstislavskiy, M. Banaru, L. Hallen et al., 2009 Transcriptome analysis by strand-specific sequencing of complementary DNA. Nucleic Acids Res. 37: e123. https://doi.org/10.1093/nar/gkp596

Rao, S. S., M. H. Huntley, N. C. Durand, E. K. Stamenova, I. D. Bochkov et al., 2014 A 3D map of the human genome at kilobase resolution reveals principles of chromatin looping. Cell 159: 1665-1680 [corrigenda: Cell 162: 687-688 (2015)]. https://doi.org/10.1016/j.cell.2014.11.021 
Rocha, P. P., R. Raviram, R. Bonneau, and J. A. Skok, 2015 Breaking TADs: insights into hierarchical genome organization. Epigenomics 7: 523-526. https://doi.org/10.2217/epi.15.25

Rowley, M. J., and V. G. Corces, 2016 The three-dimensional genome: principles and roles of long-distance interactions. Curr. Opin. Cell Biol. 40: 8-14. https://doi.org/10.1016/ j.ceb.2016.01.009

Rutledge, M. T., M. Russo, J. M. Belton, J. Dekker, and J. R. Broach, 2015 The yeast genome undergoes significant topological reorganization in quiescence. Nucleic Acids Res. 43: 8299-8313. https://doi.org/10.1093/nar/gkv723

Schalbetter, S. A., A. Goloborodko, G. Fudenberg, J. M. Belton, C. Miles et al., 2017 SMC complexes differentially compact mitotic chromosomes according to genomic context. Nat. Cell Biol. 19: 1071-1080. https://doi.org/10.1038/ncb3594

Sexton, T., E. Yaffe, E. Kenigsberg, F. Bantignies, B. Leblanc et al., 2012 Three-dimensional folding and functional organization principles of the Drosophila genome. Cell 148: 458-472. https:// doi.org/10.1016/j.cell.2012.01.010

Spearman, M. B. M., 2007 Animal Cell Biotechnology: Methods and Protocols. Springer Science \& Business Media, Totowa, NJ.

Sutani, T., T. Sakata, R. Nakato, K. Masuda, M. Ishibashi et al., 2015 Condensin targets and reduces unwound DNA structures associated with transcription in mitotic chromosome condensation. Nat. Commun. 6: 7815. https://doi.org/10.1038/ ncomms8815

Swygert, S. G., S. Kim, X. Wu, T. Fu, T. H. Hsieh et al., 2018 Condensin-dependent chromatin condensation represses transcription globally during quiescence. bioRxiv. Available at: https://doi.org/10.1101/320895.

Terakawa, T., S. Bisht, J. M. Eeftens, C. Dekker, C. H. Haering et al., 2017 The condensin complex is a mechanochemical motor that translocates along DNA. Science 358: 672-676. https:// doi.org/10.1126/science.aan6516

Tjong, H., K. Gong, L. Chen, and F. Alber, 2012 Physical tethering and volume exclusion determine higher-order genome organization in budding yeast. Genome Res. 22: 1295-1305. https:// doi.org/10.1101/gr.129437.111

Trapnell, C., L. Pachter, and S. L. Salzberg, 2009 TopHat: discovering splice junctions with RNA-Seq. Bioinformatics 25: 11051111. https://doi.org/10.1093/bioinformatics/btp120

Uhlmann, F., 2016 SMC complexes: from DNA to chromosomes. Nat. Rev. Mol. Cell Biol. 17: 399-412. https://doi.org/10.1038/ nrm.2016.30

Van Bortle, K., M. H. Nichols, L. Li, C. T. Ong, N. Takenaka et al., 2014 Insulator function and topological domain border strength scale with architectural protein occupancy. Genome Biol. 15: R82. https://doi.org/10.1186/gb-2014-15-5-r82

van Ruiten, M. S., and B. D. Rowland, 2018 SMC complexes: universal DNA looping machines with distinct regulators. Trends Genet. 34: 477-487. https://doi.org/10.1016/j.tig.2018.03.003

Walther, N., M. J. Hossain, A. Z. Politi, B. Koch, M. Kueblbeck et al., 2018 A quantitative map of human condensins provides new insights into mitotic chromosome architecture. J. Cell Biol. 217: 2309-2328. https://doi.org/10.1083/jcb.201801048

Wang, D., A. Mansisidor, G. Prabhakar, and A. Hochwagen, 2016 Condensin and Hmol mediate a starvation-induced transcriptional position effect within the ribosomal DNA array. Cell Rep. 14: 1010-1017 (erratum: Cell Rep. 17: 624). https:// doi.org/10.1016/j.celrep.2016.09.057

Wang, J., T. Blevins, R. Podicheti, J. R. Haag, E. H. Tan et al., 2017 Mutation of Arabidopsis SMC4 identifies condensin as a corepressor of pericentromeric transposons and conditionally expressed genes. Genes Dev. 31: 1601-1614. https://doi.org/ 10.1101/gad.301499.117

Wang, R., J. Mozziconacci, A. Bancaud, and O. Gadal, 2015 Principles of chromatin organization in yeast: relevance of polymer models to describe nuclear organization and dynamics. Curr. Opin. Cell Biol. 34: 54-60. https://doi.org/10.1016/j.ceb. 2015.04.004

Wang, X., T. B. Le, B. R. Lajoie, J. Dekker, M. T. Laub et al., 2015 Condensin promotes the juxtaposition of DNA flanking its loading site in Bacillus subtilis. Genes Dev. 29: 1661-1675. https://doi.org/10.1101/gad.265876.115

Yong-Gonzalez, V., B. D. Wang, P. Butylin, I. Ouspenski, and A. Strunnikov, 2007 Condensin function at centromere chromatin facilitates proper kinetochore tension and ensures correct mitotic segregation of sister chromatids. Genes Cells 12: 1075-1090. https://doi.org/10.1111/j.1365-2443.2007.01109.x

Yu, H. G., and D. E. Koshland, 2003 Meiotic condensin is required for proper chromosome compaction, SC assembly, and resolution of recombination-dependent chromosome linkages. J. Cell Biol. 163: 937-947. https://doi.org/10.1083/jcb.200308027

Yue, J. X., J. Li, L. Aigrain, J. Hallin, K. Persson et al., 2017 Contrasting evolutionary genome dynamics between domesticated and wild yeasts. Nat. Genet. 49: 913-924. https:// doi.org/10.1038/ng.3847

Zhang, Y., T. Liu, C. A. Meyer, J. Eeckhoute, D. S. Johnson et al., 2008 Model-based analysis of ChIP-Seq (MACS). Genome Biol. 9: R137. https://doi.org/10.1186/gb-2008-9-9-r137

Communicating editor: O. Rando 